\title{
Accelerating Face Detection by Using Depth Information
}

\author{
Haiyuan Wu, Kazumasa Suzuki, Toshikazu Wada, and Qian Chen \\ Faculty of Systems Engineering, Wakayama University 930 Sakaedani, \\ Wakayama-city, Wakayama, 640-8510 Japan \\ \{wuhy, twada, chen\}asys.wakayama-u.ac.jp, \\ suzuki@vrl.sys.wakayama-u.ac.jp
}

\begin{abstract}
In the case that the sizes of faces are not available, all possible sizes of faces have to be assumed and a face detector has to classify many (often ten or more) sub-image regions everywhere in an image. This makes the face detection slow and the high false positive rate. This paper explores the usage of depth information for accelerating the face detection and reducing the false positive rate at the same time. In detail, we use the depth information to determine the size of the sub-image region that needs to be classified for each pixel. This will reduce the number of sub-image regions that need to be classified from many to one for one position (pixel) in an image. Since most unnecessary classifications are effectively avoided, both the processing time for face detection and the possibility of false positive can be reduced greatly. We also propose a fast algorithm for estimating the depth information that is used to determine the size of sub-image regions to be classified.
\end{abstract}

Keywords: Face detection, Face size, Depth information, Stereo, Video rate.

\section{Introduction}

Face detection is a time consuming job [4][5]. This is not only because of the difficulty of classifying a sub-image region into "face" or "non-face" class, but also because of the huge number of sub-image regions to be classified. There are two possible approaches to make face detection fast. The first one is to reduce the processing time for classifying a sub-image region. The second one is to reduce the number of sub-image regions to be classified in an image.

Many methods about the first approach have been proposed, among them there is the Viola's one. They used Integral Image to computer the Haar-Like features quickly, and selected a small number of critical visual features by AdaBoost. They also combined classifiers in a cascade to discarded the background regions quickly.

In the job of face detection where the sizes and the positions of faces are not available, sub-image regions of all possible sizes at any position have to classify. Therefore, the number of sub-image regions to be classified is so many that makes the face detection slow even with a very efficient face detection algorithm.

In an experiment of detecting faces in images of $320 \times 240$ pixels using the Viola's algorithm[1] implemented in OpenCV[2], the processing time was about 70 milliseconds on a $3.0 \mathrm{GHz}$ Pentium 4 PC. In this experiment, the size of sub-image regions (windows) for detecting faces starts from $20 \times 20$ pixels and is enlarged by 1.25 time in

T. Wada, F. Huang, and S. Lin (Eds.): PSIVT 2009, LNCS 5414, pp. 657-667, 2009.

(C) Springer-Verlag Berlin Heidelberg 2009 
each step until it covers the whole image. In this case, the number of sub-image regions to be classified at the same position is

$$
\begin{aligned}
N_{\text {classification }} & =\frac{\log (\text { Biggest })-\log (\text { Smallest })}{\log (\text { Scale facteor })} \\
& =\frac{\log 240-\log 20}{\log 1.25}=11 .
\end{aligned}
$$

This indicates that the famous approach only cannot make face detection fast enough. Another problem is the difficulty of telling a real face from a face in a photograph (See figure 11.

Another necessary approach to make face detection fast is to reduce the number of sub-image regions to be classified in an image. In this paper, we describe a new method for reducing the sub-image regions to be classified for face detection. We estimate the size of face that may exist at each position in an image from the depth information. In this way we can determine the size of sub-image regions that need to be classified. Therefore the number of sub-image regions to be classified at each position can be reduced from several (often ten or more) to one. The depth information is obtained from a stereo camera while the faces are detected from one image of the stereo image pair.

Assuming the 3D size of human's heads is constant and the images are taken by a calibrated camera, the size of a face in an image can be calculated from the depth information. For each pixel in an image, if we assume that a face exists there, we can determine the size of the face from the depth at that pixel. Thus for that pixel we only need to check if the sub-image region of that size really contains a face or not.

Since most unnecessary classifications can be effectively avoided with our method, both the processing time for face detection and the possibility of false positive can be reduced greatly. We also propose a fast algorithm for estimating the depth information that is used to determine the size of sub-image regions to be classified.

Several researches related to face detection using stereo cameras have been reported. All of them are different from our method.

Darrell et al. [6] used stereo vision, color and pattern recognition to track a head. In this research, they segmented the range data into several connected regions. If a face detected with pattern recognition or by skin color detection overlaps with one of the segmented region, they say that region indicates a human body.

There are some researches about head tracking (not detecting) that only use range images. Yang et al. [7] presented a model-based stereo head tracking system. The processing is purely performed on range image; the color/grey camera images were not used. Seven landmark points on each face in the first image frame have to be selected manually for estimating the initial 3D head pose. N. Jojic[8]'s method requires manual initialization. B. Deniel[9]'s method requires that the background is modeled and can be updated.

Luo et a1 [10] used stereo camera to detect heads by subtracting the background, which requires the prior knowledge of background. Wang et a1 [11] detected heads in range image by using morphological watersheds. They separated the nearest face from other objects by analysis of the disparity histogram. They only track the face nearest to the camera. 


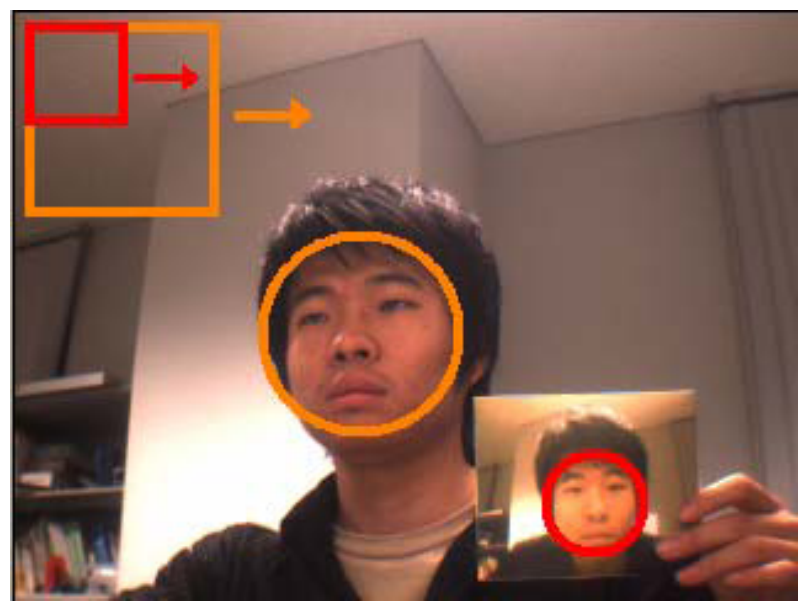

Fig. 1. An pattern recognition based face detection method can not distinguish a real face and the face in a photograph

This paper describes an efficient approach that enables video rate face detection by using depth information. We use a commercial stereo camera and the software for estimating range data provided by the camera maker. In order to complete the depth estimation within 10 millisecond, we propose a new adaptive sparse sampling method optimized for determining the size of sub-image regions to be classified called important sampling. The classifier for detecting a face in a sub-image region is the one of Viola et al[1].

\section{Video-Rate Face Detection with Stereo-Camera}

\subsection{Stereo Depth Estimation[3]}

We use the Triclops library, which is provided with the Bumblebee stereo camera made by the Point Grey Research Inc., to estimate the depth information. The correspondence between two images, which is necessary for calculating the disparity, is established with the SAD (Sum of Absolute Differences) correlation algorithm. Given a pixel $(x, y)$ in the right image, its corresponding pixel $(x+d, y)$ in the left image is determined by finding a $d_{c}$ that minimizes the $F(x, y, d)$ as follows.

$$
d_{c}=\underset{d_{\min } \leq d \leq d_{\max }}{\operatorname{argmin}} F(x, y, z),
$$

where,

$$
F(x, y, d)=\sum_{i=-m / 2}^{m / 2} \sum_{j=-m / 2}^{m / 2}\left|I_{R}(x+i, y+j)-I_{L}(x+i+d, y+j)\right|,
$$

$m$ is the mask size, $d_{\min }$ and $d_{\max }$ are the minimum and maximum disparities. $I_{R}$ and $I_{L}$ are the right and left images. 
In this research, the window size we used for stereo correlation is $9 \times 9$ pixels $(m=$ 8 ), and the surface validation filter and the edge filter are used in the depth estimation.

\subsection{Accelerating Depth Estimation by Important Sampling}

Estimating dense (pixel by pixel) depth information from stereo image pairs is computationally expensive and is difficult to be performed at video rate. In this research, since the depth information is used for predicting the sizes of faces appeared in the image, one depth is enough for one region which containing a face. Therefore, the required depth information is sparse so that the time for depth estimation can be greatly reduced.

In this paper, we propose a novel method for adjusting the sampling interval adaptively for face detection so that the required depth information can be estimated within 10 millisecond for stereo images of $320 \times 240$ pixels.

Sparse Depth Estimation. Since the smallest detectable face is $20 \times 20$ pixels, we let the sampling interval be 20 pixels as shown in Figure 2 so that there will be at least one sample point on any detectable faces. However, the sample points are still too many for the computer to process within 10 milliseconds. In order to solve this problem, we divide the sample points into two groups (the red and blue group in Figure 2), and estimate the depths of each group alternately. This ensures that the depth of any sample point will be estimated for every two frames.

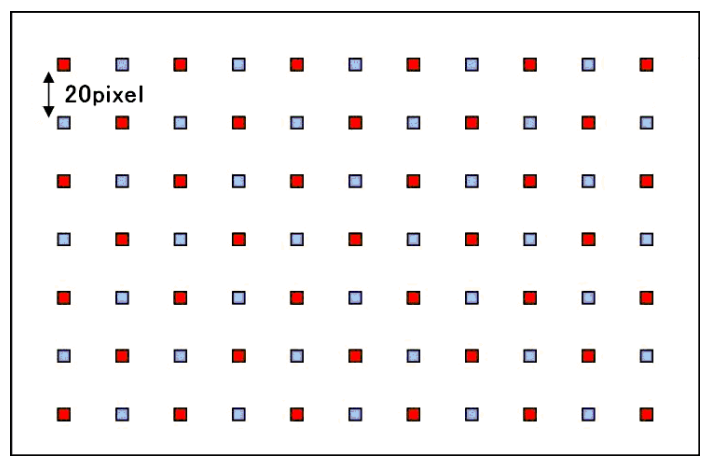

Fig. 2. Arrangement of sample points

There are two problems in this sampling method. For small faces, since the depths of the red sample points and blue sample points are estimated alternately, the depth information may not be available for every frame. And for big faces, multiple depth information will be estimated for a face which is redundancy.

Important Sampling. Since faces in video sequences move continuously, if they have been detected in an image frame, in the next frame they should be near where they have been detected. According to this fact, for each face detected in the previous frame, we set $4 \times 4$ sample points on a double sized square centered at the center of this ensures that 


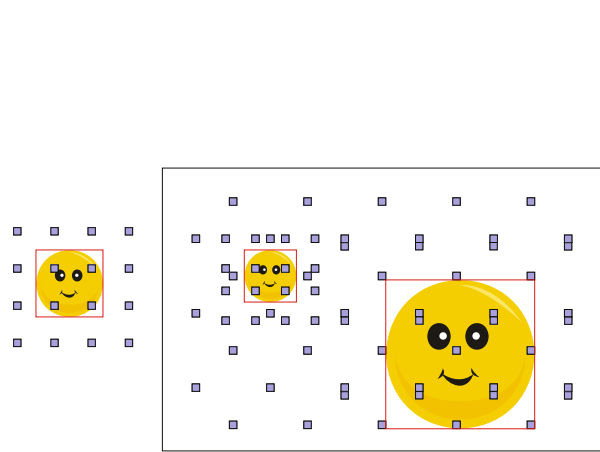

(a) Important sampling

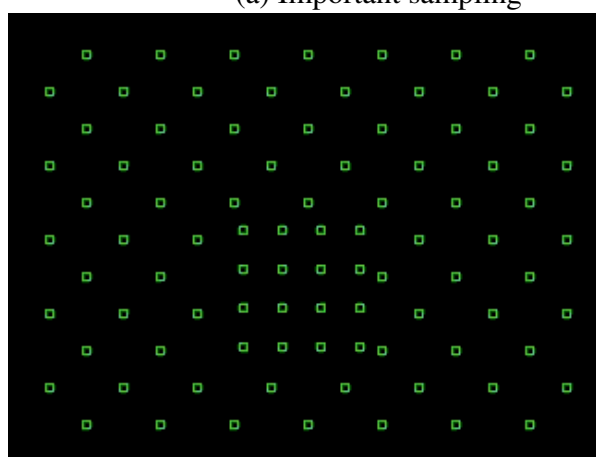

(c) Sample points of (b)

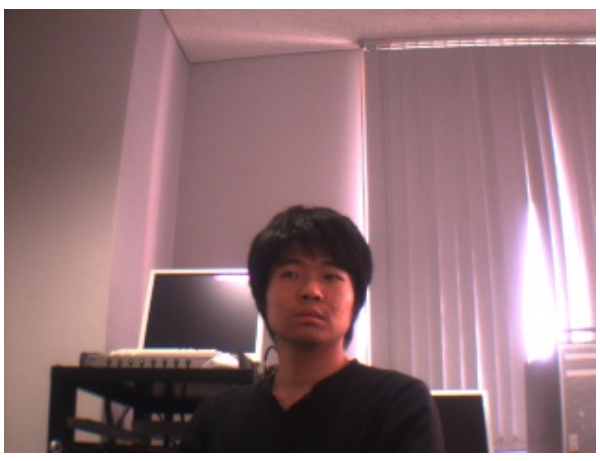

(b) Input image

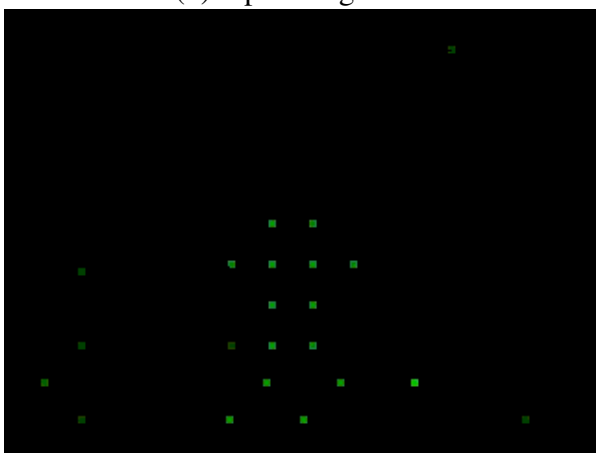

(d) Estimated depth

Fig. 3. Important sampling

the depth information of previously detected faces will be available for every frame and the sampling interval is adjusted adaptively. We call this sampling strategy as important sampling. Figure 3(b) shows an input image, and Figure 3(c) shows the arrangement of sample points for the input image. Figure 3 d) shows the estimated depths where the brightness of green dots indicates the depth (the near the brighter), while the sample points where the estimated face sizes that are too big or too small are not shown.

\subsection{Depth Based Classification Reduction}

The relation between the distance from a face to a camera (the depth $Z$ ), the width of the face in the image $\left(I_{\text {size }}\right)$ and the diameter of the face $\left(W_{\text {size }}\right)$ can be described (see figure 4) as follows.

$$
I_{\text {size }}=f \frac{W_{\text {size }}}{Z},
$$

where $f$ is the focal length of the camera. Since the size of human head is almost the same, if a sample point is on a face in the image, the face width can be calculated from the depth with eq.(2). This indicates that we only need to check if the sub-image region of the estimated face size contains a face. 


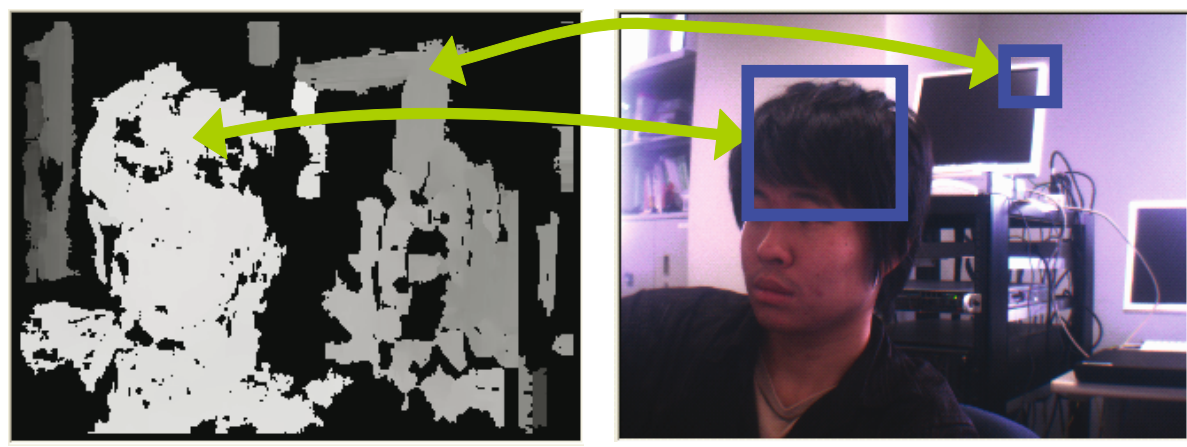

Fig. 4. Estimating the face width in image using depth information

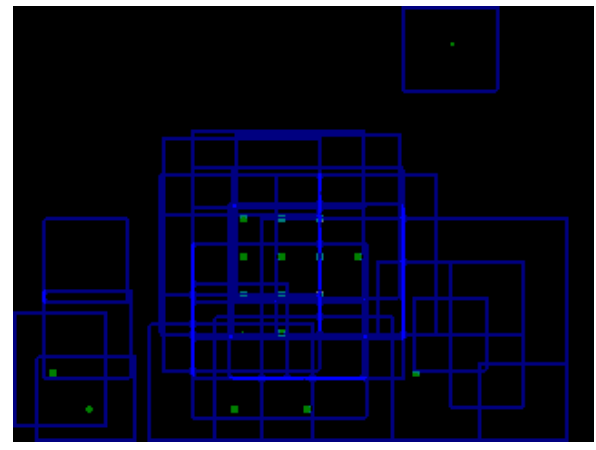

(a)

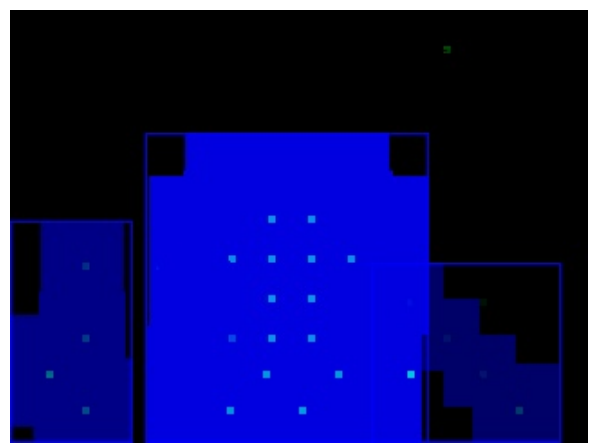

(b)

Fig. 5. Integration of overlapped image regions for face detection

If a sample point is on a face, the image region where the face may exist will be a square centered at the sample point and is double width of the estimated face width. Since the width of the square is much longer than the interval between two sample points, there may be many image regions that overlap each other. The multiple classifications for those overlapped regions are a waste of time thus should be avoided. For this purpose, we integrate the overlapped image regions where the estimated face sizes are almost same into one big rectangular image region. The face classifier is applied to that image region by using the estimated face width as the window size. Figure 5 (a) and (b) shows an example of determined image regions for face detection from all sample points and the ones after the integration.

\section{Experimentation [12]}

\subsection{Experimental Environment}

We used a PC with a 3.0GHz Pentium 4(R) CPU, a Bumblebee Stereo Vision Camera made by Point Grey Research Inc. and its supported software in the experiments. We set 
$W_{\text {size }}$ in eq.(2) to $14 \mathrm{~cm}$. Since the diameter of a head is not exactly same for different person and the estimated depth may contain some error, we tolerant the restriction of face size by using two different window size simultaneously: $I_{\text {size }}$ and $1.25 I_{\text {size }}$.

\subsection{Comparison of Processing Speed}

We applied our method and Viola's algorithm[1] to two stereo image sequences $(320 \times$ 240 pixels). In sequence 1 there is one person and in the sequence 2 there are two persons. Each of them contains about 100 frames.

Figure 6 and figure 7 show the processing time of our method for sequence 1 and 2 , respectively. The horizontal and vertical axis indicates the frame number and the processing time in millisecond, respectively. The green, orange and blue curve show the face classification time, the depth estimation time, and the sum time of our method, respectively.

Comparing figure 6 and figure 7 we noticed that the time for depth estimation (orange curve) was almost constant, while the total processing time (blue curve) for sequence 2 was longer than the one for sequence 1 . The reason is that since there are two faces in sequence 2, the image regions that need to be classified are bigger than the ones for sequence 1 . In both cases the face detection for one frame was finished in 33 milliseconds.

We also tested our method with various image sequences and confirmed that the average processing time was about 25 milliseconds for one frame. On the other hand, Viola's algorithm[1] needed about 70 milliseconds for one frame of image.

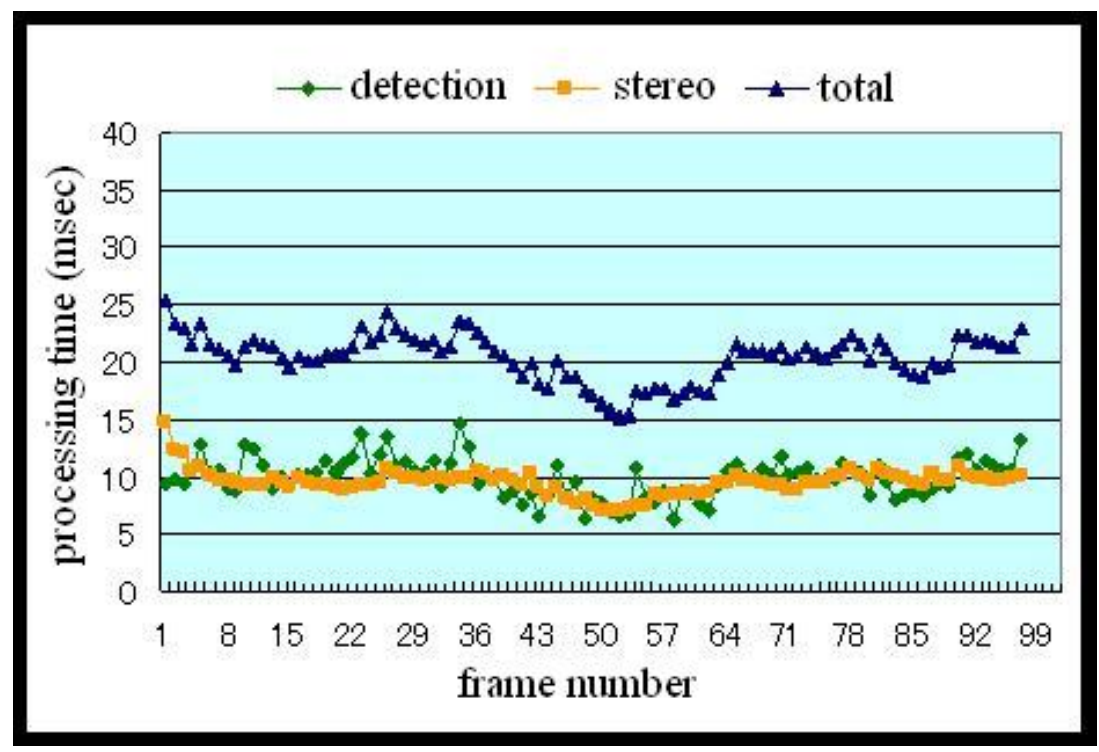

Fig. 6. The processing time of our method for sequence 1 using our method 


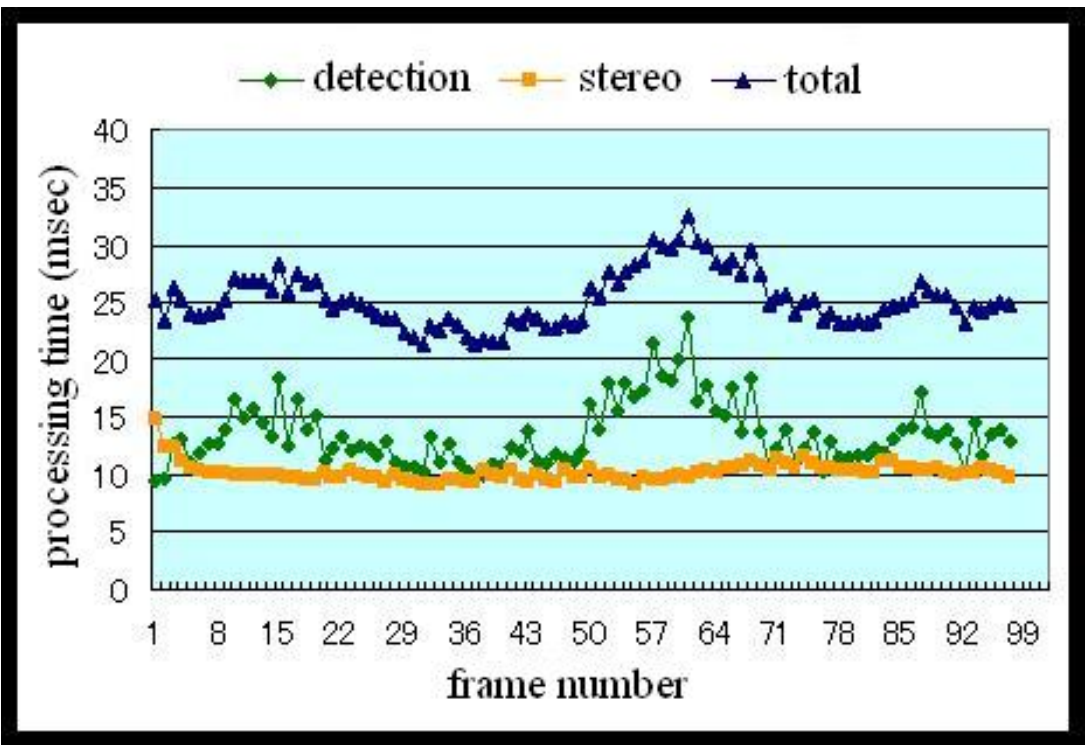

Fig. 7. The processing time of our method for sequence 2 using our method

\subsection{Comparison of Detection Accuracy}

In order to confirm the detection accuracy of the proposal method, three stereo image sequences containing 101 frames $(320 \times 240$ pixels) were prepared. There is one face in the sequences 1 and 2 , and are two faces in sequence 3 , while the face in sequence 2 moves faster.

For each sequence we conducted two different experiments as follows.

- Experiment 1: Detect faces with Viola's algorithm[1] for monocular images (using the right image of a stereo pair)

- Experiment 2: Detect faces with our method in the right image of a stereo pair.

In experiment 1 , the number of windows of different size for classification was $(\log 240-\log 20) / \log 1.25=11$. In experiment 2 , we used two window sizes for classification: Isize and 1.25Isize.

The results are summarized in Table 1 and Table 2 In sequence 3, the results were showed separately in $3 \mathrm{a}$ and $3 \mathrm{~b}$ for each face.

Table 1. The experimental results of face detection. The numbers show the total frames where the faces were successfully detected.

\begin{tabular}{|c|c|c|c|c|}
\hline Series No. & 1 & 2 & $3 \mathrm{a}$ & $3 \mathrm{~b}$ \\
\hline Experiment 1 & 82 & 52 & 54 & 83 \\
\hline Experiment 2 & 87 & 77 & 55 & 92 \\
\hline
\end{tabular}


Table 2. The experimental results of false positive. The numbers are false detection in the total frames.

\begin{tabular}{|c|c|c|c|c|}
\hline Series No. & 1 & 2 & $3 \mathrm{a}$ & $3 \mathrm{~b}$ \\
\hline Experiment 1 & 8 & 20 & 22 & 22 \\
\hline Experiment 2 & 2 & 7 & 2 & 2 \\
\hline
\end{tabular}

From table 1 comparing the results of experiment 1 and 2, we noticed that the detection rate of our method was higher than Viola's algorithm. One considerable reason is that the window size in our method was fine fitting to the actual face, thus more reliable results can be obtained.

From table 2, we confirmed our method had much less false positive than Viola's algorithm[1].

\subsection{Other Detection Results}

Detecting Real Human Face. An example frame in a video sequence is shown in Figure 8. There is a real human face and a photograph of a face in the input image. As shown in Figure 8(a), both the real human face and the face in the photograph were detected by the viola's method. As shown in Figure 8 b), only the real human face was detected by our method. This is because our method estimates the window size of a face in input image from the stereo depth information. If the size of a face appeared in an image is much different from the size estimated from the depth information, it will not be considered as a real human face.

Detecting Rotated Faces. Since the time for face detection can be significantly reduced by using the proposed method, we can use the saved time to rotate the image so that the faces with rotation in the image plane will also become detectable.

We performed several experiments where we rotated the input images to left and right by 30 degrees, then we applied the our method to detect face in the original image and

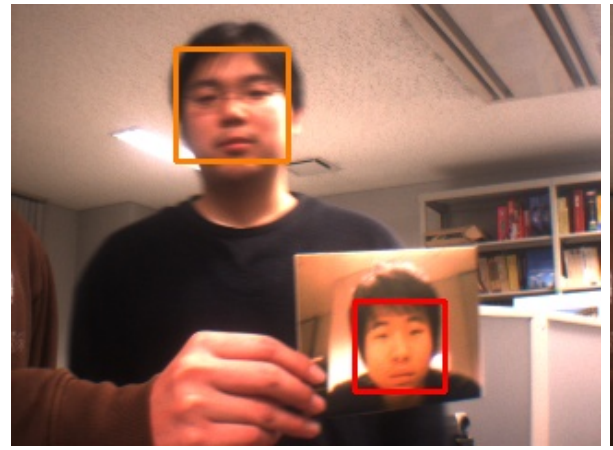

(a) Viola's method;

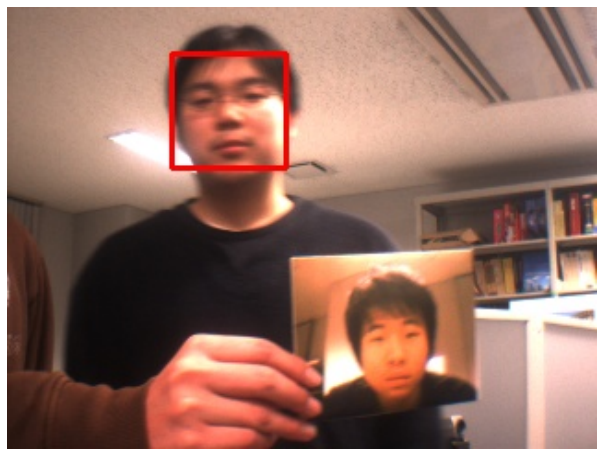

(b) Our method.

Fig. 8. A example result of detecting real human face 


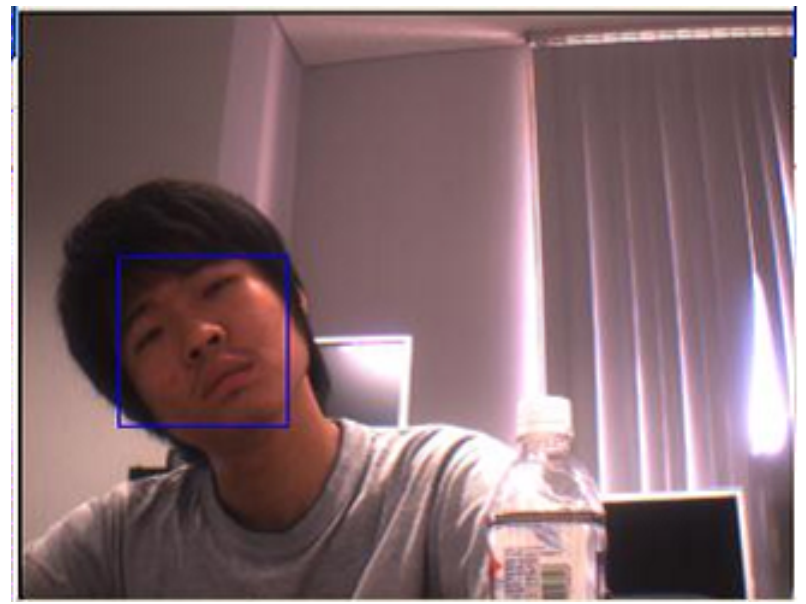

Fig. 9. A example result of detecting a face with rotation

the two rotated images. An example of the face detection result is shown in Figure 9 The processing time of these experiments was about 40-50 milliseconds per frame.

\section{Conclusion}

In this paper, we have proposed a novel method for accelerating face detection by reducing the number of sub-image regions that need to be classified. This is carried out by estimating the possible face size from the depth information obtained from a stereo camera. This has the significant effects of reducing the number of classification and suppressing the false positive rate. Moreover, we have proposed a novel method for estimating the depth information efficiently for face detection called important sampling.

Extensive experiments using various real image sequences showed that the processing time was short enough for video rate face detection. Also we have confirmed that our method can improve the detection accuracy and can decrease the false positive compared with the conventional algorithm[1].

\section{References}

1. Viola, P., Jones, M.: Robust Real-Time Face Detection. IJCV 57(2), 137-154 (2004)

2. OpenCV, http://www. intel.com/technology/computing/opencv

3. StereoVisionProducts, http://www.ptgrey.com/products/triclopsSDK/index.asp

4. Hjelmas, E.: Face detection: A survey. Computer Vision and Image Understanding 83, 236-274 (2001)

5. Yang, M.-H., Kriegman, D.J., Ahuja, N.: Detecting faces in images: a survey. PAMI 24(1), 34-58 (2002)

6. Damell, T., Gordon, G., Woodfill, J., Harville, M.: Integrated person tracking using stereo, color and pattern detection. IJCV, 175-185 (2000) 
7. Yang, R., Zhang, Z.: Model-based head tracking with stereo vision, FG, pp. 255-260 (2002)

8. Jojic, N., Turk, M., Huang, T.: Tracking self occlude articulated objects in dense disparity maps. ICCV, 123-130 (1999)

9. Deniel, B., Herman, M.: Head tracking using stereo. Machine Vision and Applications 13, 164-173 (2002)

10. Luo, R., Guo, Y.: Real-time stereo tracking of multiple moving heads. In: Proceedings of International Workshop on Recognition, Analysis and Tracking of Faces and Gestures in Real-time Systems, pp. 62-67 (2001)

11. Wang, J., Lim, E., Venkateswarlu, R.: Stereo Head/Face Detection and Tracking. ICIP, 605608 (2004)

12. Suzuki, K., Oike, H., Wu, H., Wada, T.: Video-rate face detection and tracking using active stereo-camera. In: PSIVT 2009 Demo (2009) 